\title{
Web bases for the general linear groups
}

\author{
Bruce W. Westbury
}

Received: 30 November 2010 / Accepted: 26 April 2011 / Published online: 24 May 2011

(C) Springer Science+Business Media, LLC 2011

\begin{abstract}
Let $V$ be the representation of the quantized enveloping algebra of $\mathfrak{g l}(n)$ which is the $q$-analogue of the vector representation and let $V^{*}$ be the dual representation. We construct a basis for $\bigotimes^{r}\left(V \oplus V^{*}\right)$ with favorable properties similar to those of Lusztig's dual canonical basis. In particular our basis is invariant under the bar involution and contains a basis for the subspace of invariant tensors.
\end{abstract}

Keywords Schur-Weyl duality · Invariant tensors

\section{Introduction}

The aim of this paper is to study the tensor products of the tensor products of copies of the vector representation and its dual for general linear Lie algebras and their quantized enveloping algebras from the diagram point of view.

The background to this paper is a broad program; namely, given a pivotal category find a finite presentation. This problem is stated in [13, Sect. 10], [19], [22, Problem 12.18], [12, Appendix B], [4, 20]. The pivotal categories we will consider are all spherical categories. These were introduced in [1]. Heuristically, these are an abstraction of categories of representations where tensor products and duals of representations are defined.

More precisely, given some set of vertices there is a pivotal category whose morphisms are diagrams that are planar graphs with vertices from the prescribed set. This is regarded as the free pivotal category on the vertices and the construction can be interpreted as a left adjoint. The problem then is firstly to find a finitely generated diagram category with a surjective pivotal functor to the given category and secondly

B.W. Westbury (凶)

Mathematics Institute, University of Warwick, Coventry CV4 7AL, UK

e-mail: Bruce.Westbury@warwick.ac.uk 
to find defining relations. This is analogous to the problem of finding a finite presentation for an algebra with vertices corresponding to generators and diagrams to words in the generators. There are two sources of pivotal categories which have been studied from this point of view: one is representation theory and the other is subfactors. In representation theory, the category of finite dimensional representations of a Hopf algebra is a pivotal category. A subfactor gives a planar algebra which can be regarded as a spherical category.

In this paper, we restrict our attention to strict spherical categories associated to the general and special linear Lie algebras. The basic tensors are the tensors in $[11,14,19,21]$.

The aim of the paper is to construct a basis of $\otimes^{r}\left(V \oplus V^{*}\right)$ for each $r>0$ where $V$ is the vector representation and $V^{*}$ the dual representation. The intention is that this basis should be compared with Lusztig's dual canonical basis. The basic property of this basis is that the change of basis matrix to the tensor product basis is triangular and preserves the weight. In particular, the regular representation of the Hecke algebra is a weight space of $\otimes^{r} V$, so we have a basis of the Hecke algebra. Moreover taking the subset of our basis corresponding to the zero weight space, we obtain a basis for the space of invariant tensors. A further property of our basis is that it is invariant under the involution $q \leftrightarrow q^{-1}$.

Two noteworthy properties of both this basis and the dual canonical basis is that they are cellular bases as discussed in [29] and they are both invariant under rotations in the following sense. Let $a, b$ be objects in a pivotal category. Then we have a natural isomorphism $\operatorname{Inv}\left(b \otimes a^{*}\right) \cong \operatorname{Inv}\left(a^{*} \otimes b\right)$ since both spaces are naturally isomorphic to $\operatorname{Hom}(a, b)$. This isomorphism corresponds to a bijection between the two bases.

The relevance to the problem of finding a presentation is, first, that this shows that the basic tensors are generators in the sense that the functor from the diagram category to the representation category is surjective and, second, that it gives the irreducible diagrams.

In [24], Rhoades proved an amazing cyclic sieving result about rectangular Young tableaux under the action of promotion. This result is discussed in Sect. 7 of the survey [25] on the cyclic sieving phenomenon. The paper [23] gives a simpler proof of this result in the cases $n=2$ and $n=3$ using diagrams. The paper [30] gives a simpler proof of this result for all $n$ using Lusztig's dual canonical basis. The proof in [30] can be made almost elementary by replacing the dual canonical basis with the basis constructed below. This essentially extends the method in [23] to all $n$.

\section{Quantum groups}

In this section, we give presentations for the quantized enveloping algebras of the general and special linear Lie algebras. Our basic reference for this material is Lusztig's book [17]. Then we construct the $q$-analogues of the exterior powers of the natural representations. Then we show that the direct sum of these representations is both an algebra and a coalgebra. This defines the basic tensors that we will use. 
2.1 General linear Lie algebras

Definition 2.1 The quantum group $U_{q}(\mathfrak{g l}(n))$ is the $\mathbb{Q}(q)$-algebra with generators $E_{i}, F_{i}$ for $1 \leq i \leq n-1$ and $K_{i}, K_{i}^{-1}$ for $1 \leq i \leq n$. The defining relations are

$$
\begin{aligned}
& K_{i} K_{i}^{-1}=1, \quad K_{i}^{-1} K_{i}=1, \quad K_{i}^{ \pm 1} K_{j}^{ \pm 1}=K_{j}^{ \pm 1} K_{i}^{ \pm 1}, \\
& E_{i} K_{j}= \begin{cases}q K_{j} E_{i} & \text { if } j=i, \\
q^{-1} K_{j} E_{i} & \text { if } j=i+1, \\
E_{j} K_{i} & \text { otherwise, }\end{cases} \\
& K_{i} F_{j}= \begin{cases}q{ }^{-1} F_{j} K_{i} & \text { if } i=j, \\
q F_{j} K_{i} & \text { if }|i-j|=1, \\
F_{j} K_{i} & \text { otherwise, }\end{cases} \\
& E_{i} F_{j}-F_{j} E_{i}=\delta_{i j} \frac{K_{i} K_{i+1}^{-1}-K_{i}^{-1} K_{i+1}}{q-q^{-1}}, \\
& E_{i} E_{j}=E_{j} E_{i}, \quad F_{i} F_{j}=F_{j} F_{i} \text { for }|i-j| \geq 2, \\
& E_{i}^{2} E_{j}-[2] E_{i} E_{j} E_{i}+E_{j} E_{i}^{2}=0 \text { for }|i-j|=1, \\
& F_{i}^{2} F_{j}-[2] F_{i} F_{j} F_{i}+F_{j} F_{i}^{2}=0 \text { for }|i-j|=1 .
\end{aligned}
$$

The algebra $U_{q}(\mathfrak{g l}(n))$ is a Hopf $\mathbb{Q}(q)$-algebra. The comultiplication, $\Delta$, is defined on the generators by

$$
\begin{aligned}
\Delta\left(K_{i}\right) & =K_{i} \otimes K_{i}, \\
\Delta\left(K_{i}^{-1}\right) & =K_{i}^{-1} \otimes K_{i}^{-1}, \\
\Delta\left(E_{i}\right) & =E_{i} \otimes K_{i} K_{i+1}^{-1}+1 \otimes E_{i}, \\
\Delta\left(F_{i}\right) & =F_{i} \otimes 1+K_{i}^{-1} K_{i+1} \otimes F_{i} .
\end{aligned}
$$

The counit, $\varepsilon$, is defined on the generators by

$$
\varepsilon\left(K_{i}\right)=1, \quad \varepsilon\left(K_{i}^{-1}\right)=1, \quad \varepsilon\left(E_{i}\right)=0, \quad \varepsilon\left(F_{i}\right)=0 .
$$

The antipode, $S$, is defined on the generators by

$$
S\left(K_{i}^{ \pm 1}\right)=K_{i}^{\mp 1}, \quad S\left(E_{i}\right)=-E_{i} K_{i}^{-1} K_{i+1}, \quad S\left(F_{i}\right)=-K_{i} K_{i+1}^{-1} F_{i} .
$$

Then we also have

$$
S^{-1}\left(K_{i}^{ \pm 1}\right)=K_{i}^{\mp 1}, \quad S^{-1}\left(E_{i}\right)=-K_{i} K_{i+1}^{-1} E_{i}, \quad S^{-1}\left(F_{i}\right)=-F_{i} K_{i}^{-1} K_{i+1} .
$$

The algebra $U_{q}(\mathfrak{s l}(n))$ is the subalgebra generated by $E_{i}, F_{i}$ for $1 \leq i \leq n-1$ and $K_{i} K_{i+1}^{-1}, K_{i}^{-1} K_{i+1}$ for $1 \leq i \leq n-1$. This is also a Hopf algebra. 
The integral form of $U_{q}(\mathfrak{g l}(n))$ is the $\mathbb{Z}\left[q, q^{-1}\right]$-algebra generated by $E_{i}^{r} /[r]$ !, $F_{i}^{r} /[r]$ ! for $1 \leq i \leq n-1, r \geq 1$ and $K_{i}, K_{i}^{-1}$ for $1 \leq i \leq n$.

Similarly, the integral form of $U_{q}(\mathfrak{s l}(n))$ is the $\mathbb{Z}\left[q, q^{-1}\right]$-algebra generated by $E_{i}^{r} /[r] !, F_{i}^{r} /[r]$ ! for $1 \leq i \leq n-1, r \geq 1$ and $K_{i} K_{i+1}^{-1}, K_{i}^{-1} K_{i+1}$ for $1 \leq i \leq n-1$.

The bar involution is an involution of $\mathbb{Q}$-algebras and is determined by

$$
q \mapsto q^{-1}, \quad K_{i}^{ \pm 1} \mapsto K_{i}^{\mp 1}, \quad E_{i} \mapsto E_{i}, \quad F_{i} \mapsto F_{i} .
$$

This is an involution for $U_{q}(\mathfrak{g l}(n))$ and $U_{q}(\mathfrak{s l}(n))$ and restricts to an involution on the integral forms in both cases.

\subsection{Representations}

In this section, we construct certain representations of $U_{q}(\mathfrak{g l}(n))$ and some intertwiners. These intertwiners will be taken to be the generators of a strict spherical category. These are given in [16].

The one-dimensional representations are denoted by $\operatorname{det}_{q}^{k}$ for $k \in \mathbb{Z}$. These are defined by

$$
E_{i} \mapsto 0, \quad F_{i} \mapsto 0, \quad K_{i}^{ \pm 1} \mapsto q^{ \pm k} .
$$

Then for all $r, s \in \mathbb{Z}$ we have

$$
\operatorname{det}_{q}^{r} \otimes \operatorname{det}_{q}^{s}=\operatorname{det}_{q}^{r+s} .
$$

We also define a representation of $U_{q}(\mathfrak{g l}(n))$ on $\bigwedge V$. Let $\mathbf{n}$ be the set $\{1,2, \ldots, n\}$ and let $\bigwedge V$ be the vector space with basis $\left\{v_{I} \mid I \subseteq \mathbf{n}\right\}$. Let $I \subseteq \mathbf{n}$, assume $i, j \in \mathbf{n}$ such that $i \in I$ and $j \notin I$ then put $S_{i \rightarrow j}(I)=(I \backslash i) \cup j$ so that $j$ is substituted for $i$.

Definition 2.2 The action of $U_{q}(\mathfrak{g l}(n))$ on $\bigwedge V$ is defined by

$$
\begin{aligned}
E_{i} v_{I} & = \begin{cases}v_{S_{i+1 \rightarrow i}(I)} & \text { if } i+1 \in I \text { and } i \notin I, \\
0 & \text { otherwise, }\end{cases} \\
F_{i} v_{I} & = \begin{cases}v_{S_{i \rightarrow i+1}(I)} & \text { if } i \in I \text { and } i+1 \notin I, \\
0 & \text { otherwise, }\end{cases} \\
K_{i}^{ \pm 1} v_{I} & = \begin{cases}q^{ \pm 1} v_{I} & \text { if } i \in I, \\
v_{I} & \text { otherwise. }\end{cases}
\end{aligned}
$$

Note that $E_{i}^{2} v_{I}=0$ and $F_{i}^{2} v_{I}=0$ for $1 \leq i \leq n-1$ and all $I \subset \mathbf{n}$. Therefore, the free $\mathbb{Z}\left[q, q^{-1}\right]$-module on the set $\left\{v_{I} \mid I \subseteq \mathbf{n}\right\}$ is a representation for the integral forms.

Example 2.3 Consider the vector space $V(n)$ with basis $\left\{v_{1}, \ldots, v_{n}\right\}$. This is a left $U_{q}(\mathfrak{g l}(n))$-module where the action of the generators is given by

$$
E_{i} v_{j}= \begin{cases}v_{j-1} & \text { if } j=i+1 \\ 0 & \text { otherwise }\end{cases}
$$




$$
\begin{aligned}
F_{i} v_{j} & = \begin{cases}v_{j+1} & \text { if } j=i, \\
0 & \text { otherwise }\end{cases} \\
K_{i}^{ \pm 1} v_{j} & = \begin{cases}q^{ \pm 1} v_{j} & \text { if } j=i, \\
v_{j} & \text { otherwise }\end{cases}
\end{aligned}
$$

The vector space $\bigwedge V$ is an associative algebra. This is based on [21, Lemma 2.6]. For $I, J$ disjoint subsets of $\mathbf{n}$ put

$$
\pi(I, J)=|\{(i, j) \in I \times J \mid i>j\}| .
$$

Definition 2.4 The multiplication on $\bigwedge V$ is defined by

$$
v_{I} \otimes v_{J} \mapsto \begin{cases}(-q)^{-\pi(I, J)} v_{I \cup J} & \text { if } I \cap J=\varnothing, \\ 0 & \text { otherwise. }\end{cases}
$$

This multiplication is associative since both evaluations give

$$
v_{I} \otimes v_{J} \otimes v_{K} \mapsto \begin{cases}(-q)^{-\pi(I, J, K)} v_{I \cup J \cup K} & \text { if } I, J, K \text { are pairwise disjoint, } \\ 0 & \text { otherwise, }\end{cases}
$$

where $\pi(I, J, K)=\pi(I, J)+\pi(I, K)+\pi(J, K)$. The unit is $v_{\emptyset}$.

An alternative construction of this algebra is that it is generated by $\left\{v_{i} \mid 1 \leq i \leq\right.$ $n-1\}$ and the defining relations are

$$
v_{i} v_{j}+q v_{j} v_{i}=0 \quad \text { for } 1 \leq i<j \leq n-1 .
$$

These two structures on $\bigwedge V$ are compatible in the sense that the inclusion $\mathbb{Q}(q) \rightarrow$ $\bigwedge V$ determined by $1 \mapsto v_{\varnothing}$ and the multiplication map $\wedge V \otimes \wedge V \rightarrow \wedge V$ are both homomorphisms of representations.

The vector space $\bigwedge V$ is a coalgebra. The comultiplication $\Delta$ on $\bigwedge V$ is defined by

$$
v_{I} \mapsto \sum_{J, K}(-1)^{\pi(J, K)} q^{|J| \cdot|K|} v_{J} \otimes v_{K},
$$

where the sum is over $J, K \subset \mathbf{n}$ such that $J \cap K=\emptyset$ and $J \cup K=I$. This comultiplication is coassociative.

The counit $\bigwedge V \rightarrow \mathbb{Q}(q)$ is given by

$$
v_{I} \mapsto \begin{cases}1 & \text { if } I=\varnothing, \\ 0 & \text { otherwise }\end{cases}
$$

The counit and the comultiplication are both homomorphisms of representations. The representation $\bigwedge V$ has a decomposition

$$
\bigwedge V=\bigoplus_{p=0}^{n} V(p)
$$


where $V(p)$ has basis $\left\{v_{I}|| I \mid=p\right\}$. For $0 \leq p \leq n$, the representation $V(p)$ is irreducible. A highest weight vector is $v_{I}$ where $I=\{i \in \mathbf{n} \mid i \leq p\}$, and a lowest weight vector is $v_{I}$ where $I=\{i \in \mathbf{n} \mid i \geq n-p+1\}$.

Dually, we have a representation on $\bigwedge \bar{V}$. This is the vector space with basis $\left\{\bar{v}_{I} \mid I \subseteq \mathbf{n}\right\}$.

Definition 2.5 The action of $U_{q}(\mathfrak{g l}(n))$ on $\bigwedge \bar{V}$ is defined by

$$
\begin{aligned}
E_{i} \bar{v}_{I} & = \begin{cases}\bar{v}_{S_{i+1 \rightarrow i}(I)} & \text { if } i+1 \in I \text { and } i \notin I, \\
0 & \text { otherwise, }\end{cases} \\
F_{i} \bar{v}_{I} & = \begin{cases}\bar{v}_{S_{i \rightarrow i+1}(I)} & \text { if } i \in I \text { and } i+1 \notin I, \\
0 & \text { otherwise, }\end{cases} \\
K_{i}^{ \pm 1} \bar{v}_{I} & = \begin{cases}q^{\mp 1} \bar{v}_{I} & \text { if } i \in I, \\
\bar{v}_{I} & \text { otherwise. }\end{cases}
\end{aligned}
$$

It is convenient to put $V(-p)=\bar{V}(p)$ for $1 \leq p \leq n$. Then for all $r, s \in \mathbb{Z}$ such that $-n \leq r, s, r+s \leq n$ we have a homomorphism of $U_{q}(n)$-modules

$$
V(r) \otimes V(s) \rightarrow V(r+s)
$$

\section{Flow diagrams}

In this section, we follow $[19,21]$ and introduce the category of flow diagrams.

Definition 3.1 The monoid of objects of the category $F$ is the free monoid on the set $\{1, \overline{1}, 2, \overline{2}, \ldots\}$. The category $F$ is generated as a spherical category by morphisms

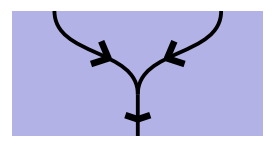

$a \otimes b \rightarrow a+b$

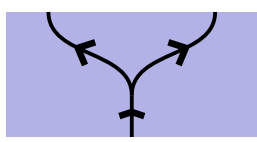

$\bar{a} \bar{b} \rightarrow \overline{a+b}$

The defining relations are the following associativity relations which hold for all labellings of the edges.
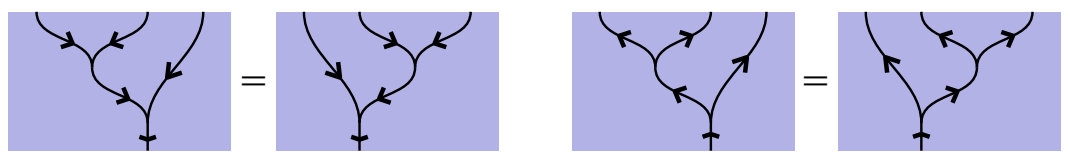

It is usually preferable to work with reduction rules rather than relations. In order to do this for the flow diagrams, it is necessary to introduce two infinite families of vertices. One family includes the first type of vertex. The number of incoming lines is arbitrary and there is one outgoing line. The condition is that the label on the outgoing 
line is the sum of the labels on the incoming line. The other family has one incoming line and an arbitrary number of outgoing lines. The condition is that the label on the incoming line is the sum of the labels on the outgoing line.

There are infinitely many reduction rules. These reduction rules are that any edge which connects two vertices in the same family can be contracted.

Next we define a functor from $F$ to the categories of representations of the integral form of $U_{q}(\mathfrak{g l}(n))$. The map of objects is determined by the map on the generators. This map is given by

$$
a \mapsto\left\{\begin{array} { l l } 
{ V ( a ) } & { \text { if } 1 \leq a \leq n , } \\
{ 0 } & { \text { if } n < a , }
\end{array} \quad \overline { a } \mapsto \left\{\begin{array}{ll}
\bar{V}(a) & \text { if } 1 \leq a \leq n, \\
0 & \text { if } n<a .
\end{array}\right.\right.
$$

The functor is defined on morphisms by defining it on the generators. These are the trivalent vertices. The images of these trivalent vertices are the intertwiners discussed in Sect. 2.2. The defining relations in $\mathrm{F}$ are satisfied since $\wedge V$ is an associative algebra and a coassociative coalgebra.

The functor from $\mathrm{F}$ to the category of representations of the integral form of $U_{q}(\mathfrak{s l}(n))$ is defined by composing with the restriction functor. An edge labeled $n$ now corresponds to the trivial representation. However, these edges cannot be simply omitted. Instead, the majority of the edge can be omitted but two stubs at the ends of the edge need to be retained. This is discussed in [19].

\section{Growth algorithm}

In this section, we give the main contribution of this paper. Let $M$ be the free monoid on the set $\{1, \overline{1}, 2, \overline{2}, \ldots\}$. Then we construct a flow diagram for each element of $M$. The analogue of this construction for the exceptional Lie group $G_{2}$ was given in [27], and the analogue for Spin(7) was given in [28].

The morphisms in $\mathrm{F}$ are trivalent graphs drawn in a rectangle. In this section, the flow diagrams are trivalent graphs drawn in a triangle. The triangles are drawn as in Fig. 1. The edge $A B$ is called the top edge of the triangle.

First, we give a triangle of length one for each element of $M$. These are the triangles
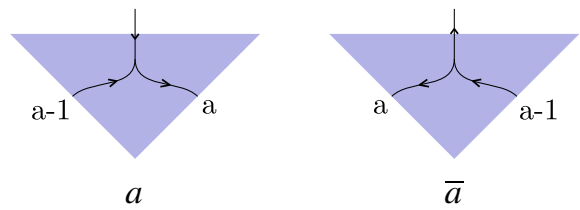

Fig. 1 Triangle

A $\mathrm{B}$ 
Then for each ordered pair of elements of $M \cup\{0\}$ we give a diamond. It is convenient to identify $M \cup\{0\}$ with $\mathbb{Z}$ by $a \mapsto a, \bar{a} \mapsto-a$ and $0 \mapsto 0$. Then we have directed edges labeled by elements of $\mathbb{Z}$. We identify a directed edge labeled $a$ with the edge with the reverse orientation and labeled $-a$. Then for each ordered pair $(a, b)$ of elements of $\mathbb{Z}$ we give a diamond. There are two cases, namely $a \neq b$ and $a=b$. The diamonds in these two cases are respectively
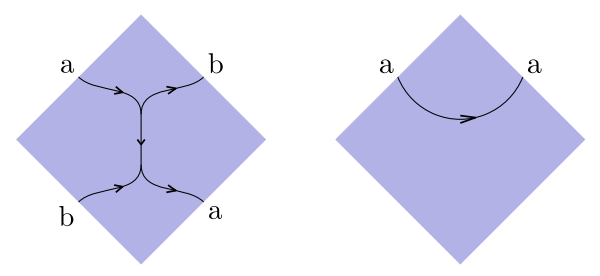

Now given a word in $M$ of length $r$, we draw a triangle of length $r$. On the top edge of this triangle, we draw the word as a sequence of triangles. Then we fill in the diamonds.

For $U_{q}(\mathfrak{g l}(n))$, flow diagrams have edges labeled by $a$ and $\bar{a}$ for $1 \leq a \leq n$. For $U_{q}(\mathfrak{s l}(n))$, the objects $\bar{a}$ and $n-a$ are isomorphic for $1 \leq a \leq n$. In particular, $n$ and $\bar{n}$ are isomorphic to the trivial representation. The cases $n=2,3,4$ are described below.

\subsection{Two part partitions}

This is the case $\mathfrak{s l}(2)$. The category of invariant tensors in this case is the TemperleyLieb category. This is studied in [26] and the account here is based on [10].

There is one type of edge which is not directed. There are no vertices.

There are two triangles which are the vertices of a crystal graph
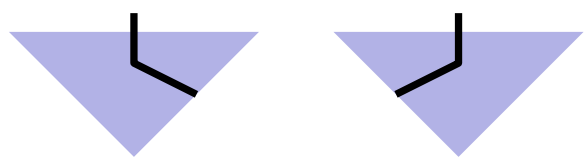

and the four diamonds
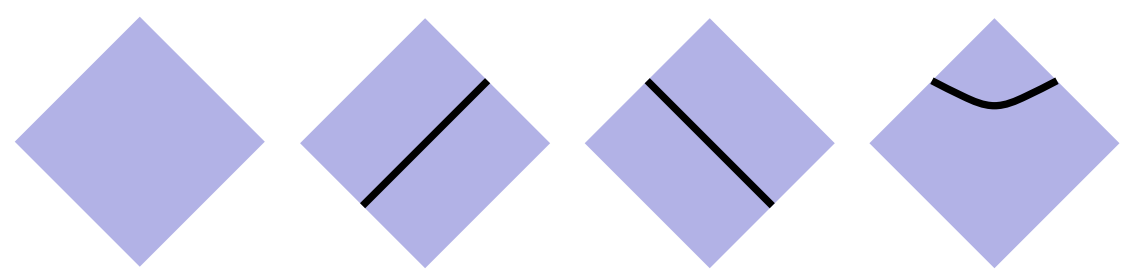
Here is an example of the growth algorithm.

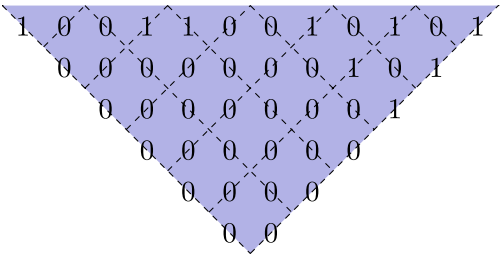

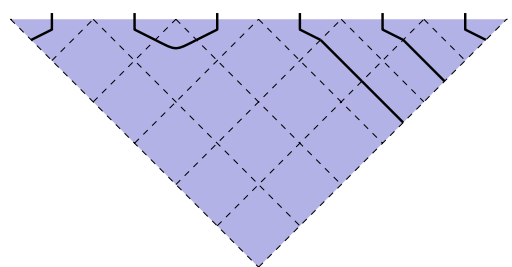

The words are words in the alphabet $\{1,2\}$. Usually, one replaces 1 by an open bracket (and 2 by a closed bracket). Then balanced lattice words are exactly wellformed parentheses.

\subsection{Three part partitions}

This is the case $\mathfrak{s l}(3)$ and is studied in [15] and [13].

There is one type of edge which is directed. There are six triangles. These are the following three together with the three obtained by reversing all directions in each of these three triangles. These are the vertices of two crystal graphs each with three vertices.
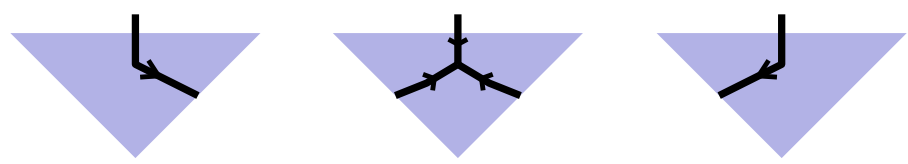

Here are the nine diamonds.
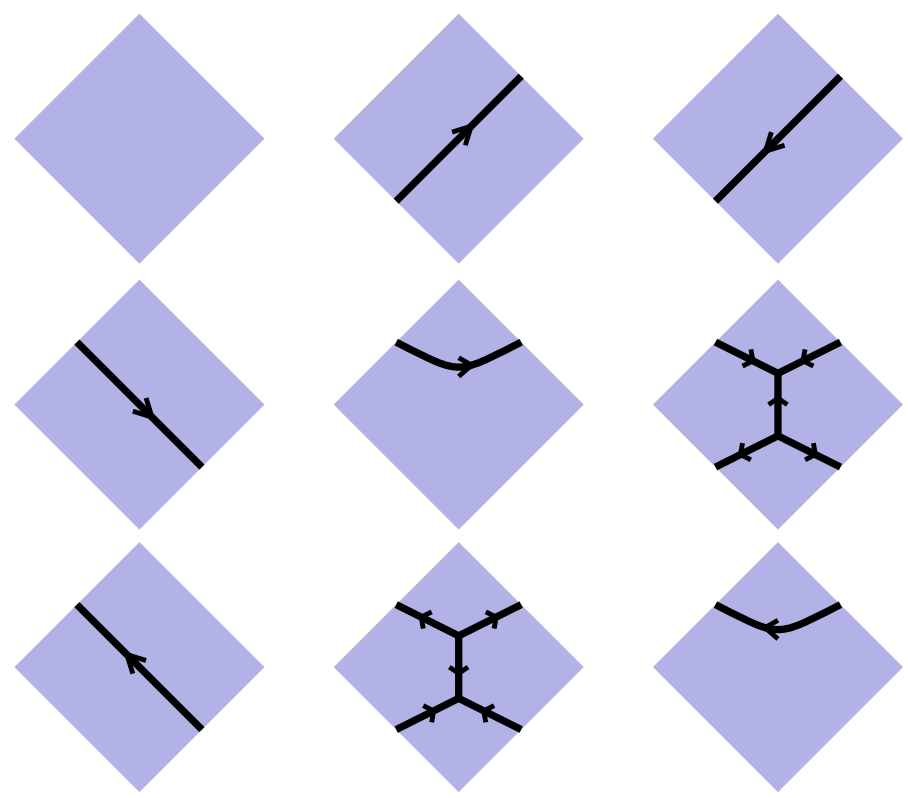
Here is an example of the growth algorithm.
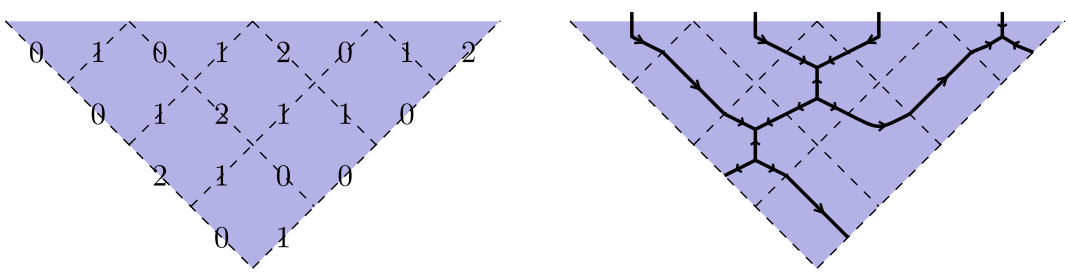

\subsection{Four part partitions}

This is the case $\mathfrak{s l}(4)$ and was studied in [14]. There are two types of an edge, one directed and one not. There are two types of a vertex. There are eight triangles. Four of these are drawn below and the other four are obtained by reversing the direction of each directed edge in each of these diagrams. These four triangles are the vertices of a crystal graph as are the other four vertices.
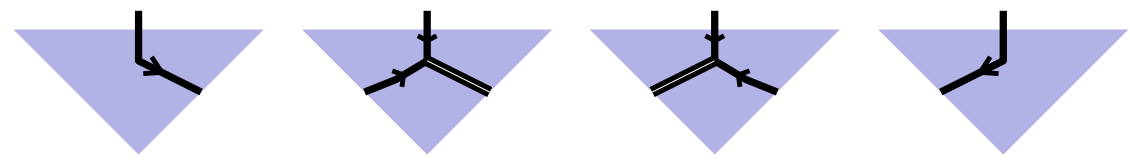

\subsection{Wave graphs}

Here we relate our growth diagrams to the wave graphs of [18]. The construction we give is an extension of the construction in this preprint.

Let $w$ be a word of length $k n$ in the alphabet $\{1,2, \ldots, n\}$. Then associated to this word are $(n-1)$ words in the alphabet $\{0,1,2\}$. For $1 \leq i \leq n-1$, let $w_{i}$ be the word obtained from $w$ by the following substitutions

$$
j \mapsto \begin{cases}1 & \text { if } j=i \\ 2 & \text { if } j=i+1 \\ 0 & \text { otherwise }\end{cases}
$$

Then it is clear that $w$ can be recovered from the words $w_{1}, \ldots, w_{n-1}$. Extend the growth algorithm in Sect. 4.1 by assigning the empty triangle to the letter 0. Apply this growth algorithm to each of the words $w_{1}, \ldots, w_{n-1}$ to get $n-1$ triangles. Now bind these pages into a book by identifying the top edges of the triangles (which forms the spine of the book). This gives the wave graph of the word $w$.

The reason for calling this a wave graph comes from the following properties of the wave graph. There are $k$ points marked on the spine. Each page of the book is a triangle with some embedded arcs. Each of these arcs either connects a point on the boundary of the page (not on the spine) with one of the marked points on the spine or connects two of the marked points on the spine. Each marked point on the spine is the endpoint of either one arc or else is the endpoint of two arcs which are on adjacent pages. 
The growth diagram of a word $w$ can be constructed directly from the wave graph of the word $w$ simply by superimposing the pages of the book. The idea here is to regard each page as transparent and then to close the book.

The special case considered in [18] is the case when each arc on every page connects two points on the spine. In this special case, each arc in the book consists of one arc from each page and has one endpoint on the first page and one endpoint on the last page. These correspond to the following:

Definition 4.1 A closed wave graph is a partition of the set $\{1,2, \ldots, k n\}$ into $k$ subsets each with $n$ elements. For $1 \leq a \leq k$ we have $i_{1}^{a}<\cdots<i_{k}^{a}$. Then the condition is that there does not exist $a$ and $r \neq s$ such that

$$
i_{r}^{a}<i_{s}^{a}<i_{r}^{a+1}<i_{s}^{a+1} .
$$

These then correspond to standard Young tableaux of shape $k^{n}$. For example, for $n=2, k=3$ we have five standard Young tableaux. These tableaux, the lattice word and the wave diagrams are

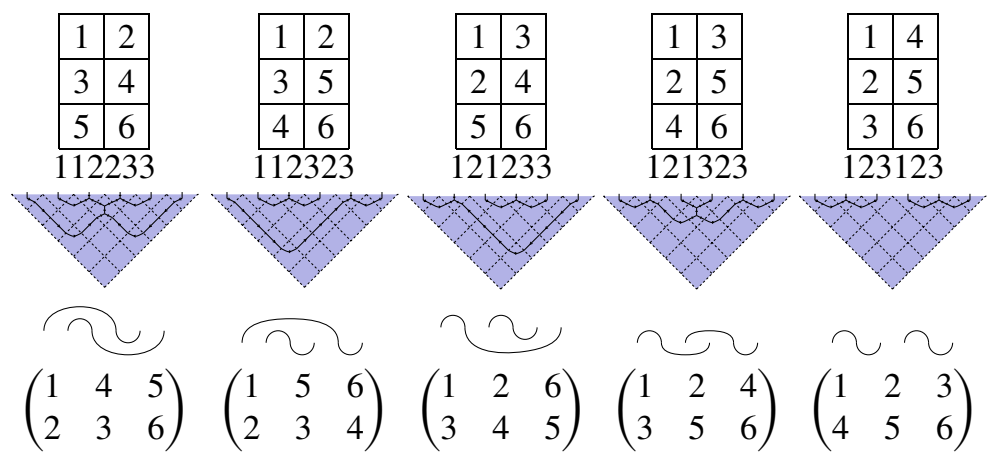

\subsection{Basis}

In Sect. 4, we defined the flow diagram of a word. In this section, we use the flow diagram of a word to construct a tensor. Then we show that these tensors are a basis of the tensor product.

Let $w$ be a word in $\{1, \overline{1}, 2, \overline{2}, \ldots, n, \bar{n}\}$. Define a type to be a word in $\{+,-\}$. The type of $w$ is the word $u$ given by the substitution $a \mapsto+, \bar{a} \mapsto-$ for $1 \leq a \leq n$. The representation $V(u)$ associated to a type $u$ is determined by the rules

$$
V(+)=V, \quad V(-)=\overline{V,} \quad V\left(u_{1} u_{2}\right)=V\left(u_{1}\right) \otimes V\left(u_{2}\right) .
$$

Let $M^{r}(n)$ be the set of words of length $r$. Then we identify this with the tensor product basis of $\bigotimes^{r}(V \oplus \bar{V})$. This tensor product has the obvious decomposition $\bigotimes^{r}(V \oplus \bar{V})=\sum_{u} V(u)$ where the sum is over types of length $r$. Then the set of words of length $r$ and type $u$ is identified with the tensor product basis of $V(u)$.

For a dominant weight $\lambda$, let $V(\lambda)$ be the corresponding irreducible highest weight representation. Define an involution $\lambda \mapsto \lambda^{*}$ by $V(\lambda)^{*} \cong V\left(\lambda^{*}\right)$. Then the lowest 
weight vector of $V\left(\lambda^{*}\right)$ has weight $-\lambda$. Let $v_{\lambda}^{\text {hi }}$ be a highest weight vector in $V(\lambda)$ and $v_{\lambda}^{\text {lo }}$ a lowest weight vector.

A flow diagram drawn in the triangle in Fig. 1 is an intertwiner

$$
\psi: V(O A) \otimes V(O B)^{*} \rightarrow V(A B)
$$

Here each edge of the triangle is a directed path which meets the flow diagram in a sequence of edges. Let the sequence of labels of these edges be $\lambda_{1}, \ldots, \lambda_{k}$. The weight of the edge is $\lambda_{1}+\cdots+\lambda_{k}$ and the associated representation is $V\left(\lambda_{1}\right) \otimes \cdots \otimes$ $V\left(\lambda_{k}\right)$.

Let $v^{\text {hi }}$ be the tensor product of the highest weight vectors in $V(O A)$ and let $v^{\text {lo }}$ be the tensor product of the lowest weight vectors in $V(O B)^{*}$. Then the element of $V(A B)$ associated to the flow diagram is $\psi\left(v^{\text {hi }} \otimes v^{\text {lo }}\right)$.

Example 4.2 The basic triangle is an intertwiner

$$
\psi: V(a) \otimes V(a-1)^{*} \rightarrow V .
$$

The associated vector in $V$ has weight $a$.

Let $w$ be a word of type $u$. The associated flow diagram is an intertwiner

$$
F(w): V(O A) \otimes V(O B)^{*} \rightarrow V(u)
$$

and this gives $A(w):=F(w)\left(v^{\mathrm{hi}} \otimes v^{\mathrm{lo}}\right) \in V(u)$. Denote the coefficients of this vector in the tensor product basis by $A_{x}^{w}$. Next we give a state sum expression for $A_{x}^{w}$.

Definition 4.3 A state assigns to each edge $e$ labeled $p$ a subset of $\{1,2, \ldots, n\}$ of size $p$. Furthermore, at each vertex we require that the subsets on the incoming edges be disjoint, that the subsets on the outgoing edges be disjoint, and that the two unions of subsets be equal.

Each state $\sigma$ has a heft $h t(\sigma) \in \mathbb{Z}\left[q, q^{-1}\right]$. The heft of a state is the product over the trivalent vertices, maxima and minima of the diagram. Note that each term in the product is a unit in $\mathbb{Z}\left[q, q^{-1}\right]$ and so for any state $\sigma, \operatorname{ht}(\sigma)$ is a unit.

The coefficients $A_{x}^{w}$ can be expressed as a state sum. Consider the flow diagram $F(w)$. The boundary edges are assigned subsets by taking the highest weight vectors on edge $O A$, lowest weight vectors on edge $O B$, and taking the sequence of singletons on edge $A B$ to be the word $x$.

Example 4.4 The basic triangles are labeled

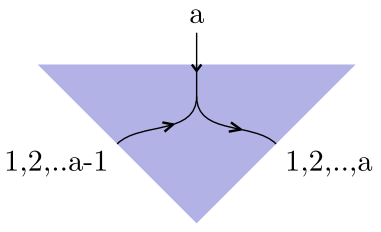

$a$

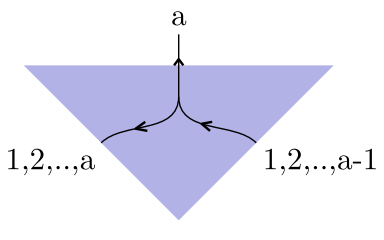

$\bar{a}$ 
Then we have

$$
A_{x}^{w}=\sum_{\sigma} \operatorname{ht}(\sigma)
$$

where the sum is over all states which satisfy the boundary conditions.

Theorem 4.5 For all $r \geq 0$, the set $\left\{A(w) \mid w \in M^{r}\right\}$ is a basis of $\bigotimes^{r}(V \oplus \bar{V})$.

Proof This is equivalent to the statement that the matrix $A$ is invertible. Let $\leq$ be the lexicographic order on $M^{r}$. It follows from (2) that if $A_{x}^{w} \neq 0$ then $x \leq w$ and $A_{w}^{w} \in$ $\mathbb{Z}\left[q, q^{-1}\right]$ is a unit. This means $A$ is a triangular matrix with units on the diagonal and so is invertible.

The reason $A_{w}^{w}$ is a unit is that the sum (2) only has one term. This is the state in which the states of every diamond are given by

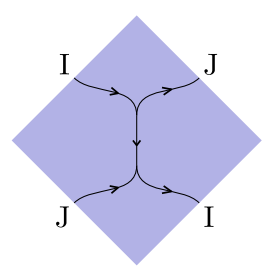

Denote this basis by $B^{r}$. For each type $u$ of length $r$ put

$$
B(u)=\{\psi(w) \mid w \text { has type } u\} .
$$

Then $B(u)$ is a basis of $V(u)$.

The weight of a word $w$ is the vector $\lambda(w)=\left(\lambda_{1}, \ldots, \lambda_{n}\right)$. Write \#a for the number of occurrences of the letter $a$ in $w$. Then $\lambda_{a}=\# a-\# \bar{a}$ for $1 \leq a \leq n$.

There are two weights associated to a flow diagram. One is $H$ which is the weight of the edge $O A$ and the other is $D$ which is the weight of the edge $O B$. For the flow diagram of $w$, we have $\lambda(w)=H-D$. Furthermore, for every state $\sigma$ of $F(w)$ the weight of $w(\sigma)$ is the weight of $w$.

Corollary 4.6 For each type $u$, the set

$$
\{A(w) \mid H(w)=0, D(w)=0\}
$$

is a basis of the invariant tensors in $V(u)$.

The case $u=+^{k}$ is [18, Theorem 1].

\section{Applications}

The first applications are to Schur-Weyl duality and extensions. For any $u$, the vector space $\operatorname{End}(V(u))$ can be identified with the invariant tensors in $V(u) \otimes V(u)^{*}$. 
Then, by Corollary 4.6, we have constructed a basis of this algebra. This approach is characteristic-free. For $u=+^{r}$, this gives the original Schur-Weyl duality, see [9]. For $u=+{ }^{r}-^{s}$, End $(V(u))$ is a $q$-analogue of the walled Brauer algebra. The walled Brauer algebra is studied in [5], and the $q$-analogue is studied in [6] and [7]. For $u=(+-)^{r}$, we have the derangement algebras studied in [3] and [2]. These versions of Schur-Weyl duality over $\mathbb{C}$ are discussed in [8].

The main open problem is the problem of finding defining relations. In a theoretical sense, we have solved this problem. Given any flow diagram, we can expand it in terms of the tensor product basis and then change basis to the basis of flow diagrams. Unfortunately, this is not useful, and it is an open problem to find a more effective algorithm for writing a general flow diagram in this basis. Defining relations for SL(2) are well known, defining relations for SL(3) are given in [15], relations for SL(4) are given in [14] with the conjecture that these are defining relations, and relations for all $\mathrm{SL}(n)$ are given in [19].

As a first step towards this, we present an algorithm which decides if a given flow diagram is an element of the basis. First, we construct a word from the flow diagram using cut paths and then we construct the flow diagram from the word. The original flow diagram is a basis vector if and only if these two flow diagrams are the same.

\section{References}

1. Barrett, J.W., Westbury, B.W.: Spherical categories. Adv. Math. 143(2), 357-375 (1999)

2. Benkart, G., Chakrabarti, M., Halverson, T., Leduc, R., Lee, C., Stroomer, J.: Tensor product representations of general linear groups and their connections with Brauer algebras. J. Algebra 166(3), 529-567 (1994)

3. Benkart, G., Doty, S.: Derangements and tensor powers of adjoint modules for $\mathfrak{s l}_{n}$. J. Algebr. Comb. 16(1), 31-42 (2002)

4. Bigelow, S.: Skein theory for the $A D E$ planar algebras. J. Pure Appl. Algebra 214(5), 658-666 (2010)

5. Cox, A., De Visscher, M., Doty, S., Martin, P.: On the blocks of the walled Brauer algebra. J. Algebra 320(1), 169-212 (2008)

6. Dipper, R., Doty, S., Stoll, F.: Quantized mixed tensor space and Schur-Weyl duality I, 2009

7. Dipper, R., Doty, S., Stoll, F.: Quantized mixed tensor space and Schur-Weyl duality II, 2009

8. Doty, S.: New versions of Schur-Weyl duality. In: Finite Groups 2003, pp. 59-71. Walter de Gruyter, Berlin (2004)

9. Doty, S.: Schur-Weyl duality in positive characteristic. In: Representation Theory. Contemp. Math., vol. 478, pp. 15-28. Am. Math. Soc., Providence (2009)

10. Frenkel, I.B., Khovanov, M.G.: Canonical bases in tensor products and graphical calculus for $U_{q}\left(\mathfrak{s l}_{2}\right)$. Duke Math. J. 87(3), 409-480 (1997)

11. Jeong, M.-J., Kim, D.: Quantum $\mathfrak{s l}(n, \mathbb{C})$ link invariants. arXiv:math.GT/0506403

12. Jones, V.F.R.: The annular structure of subfactors. In: Essays on geometry and related topics, Vols. 1, 2. Monogr. Enseign. Math., vol. 38, pp. 401-463. Enseignement Math., Geneva (2001)

13. Khovanov, M., Kuperberg, G.: Web bases for sl(3) are not dual canonical. Pac. J. Math. 188(1), 129153 (1999)

14. Kim, D.: Graphical Calculus on Representations of Quantum Lie Algebras. Ph.D. thesis, University of California, Davis. ProQuest LLC, Ann Arbor (2003). arXiv:math.QA/0310143

15. Kuperberg, G.: Spiders for rank 2 Lie algebras. Commun. Math. Phys. 180(1), 109-151 (1996). arXiv:q-alg/9712003

16. Leclerc, B., Thibon, J.-Y.: The Robinson-Schensted correspondence, crystal bases, and the quantum straightening at $q=0$. Electron. J. Comb. 3(2), 11 (1996)

17. Lusztig, G.: Introduction to Quantum Groups. Progress in Mathematics, vol. 110. Birkhäuser Boston, Boston (1993) 
18. Mihailovs, A.: Tensor invariants of SL(n), wave graphs and L-tris. arXiv:math.RT/9802119

19. Morrison, S.: A diagrammatic category for the representation theory of U_q(sl_n). PhD thesis, University of California, Berkeley, 2007

20. Morrison, S., Peters, E., Snyder, N.: Skein theory for the $D_{2 n}$ planar algebras. J. Pure Appl. Algebra 214(2), 117-139 (2010)

21. Murakami, H., Ohtsuki, T., Yamada, S.: Homfly polynomial via an invariant of colored plane graphs. Enseign. Math. 44(3-4), 325-360 (1998)

22. Ohtsuki, T.: Problems on invariants of knots and 3-manifolds. In: Invariants of Knots and 3-Manifolds, Kyoto, 2001. Geom. Topol. Monogr., vol. 4, pp. 377-572. Geom. Topol. Publ., Coventry (2002). With an introduction by J. Roberts, pp. i-iv

23. Petersen, T.K., Pylyavskyy, P., Rhoades, B.: Promotion and cyclic sieving via webs. J. Algebr. Comb. 30(1), 19-41 (2009)

24. Rhoades, B.: Cyclic sieving, promotion, and representation theory. J. Comb. Theory, Ser. A 117(1), 38-76 (2010)

25. Sagan, B.: The cyclic sieving phenomenon: a survey, 2010

26. Westbury, B.W.: The representation theory of the Temperley-Lieb algebras. Math. Z. 219(4), 539-565 (1995)

27. Westbury, B.W.: Enumeration of non-positive planar trivalent graphs. J. Algebr. Comb. 25(4), 357373 (2007)

28. Westbury, B.W.: Invariant tensors for the spin representation of $\mathfrak{s o ( 7 ) . ~ M a t h . ~ P r o c . ~ C a m b . ~ P h i l o s . ~ S o c . ~}$ 144(1), 217-240 (2008)

29. Westbury, B.W.: Invariant tensors and cellular categories. J. Algebra 321(11), 3563-3567 (2009)

30. Westbury, B.W.: Invariant tensors and the cyclic sieving phenomenon. Electron. J. Comb. (2011, in press) 